\title{
Brightness limitations of cold field emitters caused by Coulomb interactions
}

\author{
B. Cook, ${ }^{\text {a) }}$ T. Verduin, C. W. Hagen, and P. Kruit \\ Delft University of Technology, Lorentzweg 1, 2628 CJ Delft, The Netherlands
}

(Received 14 July 2010; accepted 20 September 2010; published 1 December 2010)

\begin{abstract}
Emission theory predicts that high brightness cold field emitters can enhance imaging in the electron microscope. This (neglecting chromatic aberration) is because of the large (coherent) probe current available from a high brightness source and is based on theoretically determined values of reduced brightnesses up to $10^{14} \mathrm{~A} /\left(\mathrm{m}^{2} \mathrm{sr} \mathrm{V}\right)$. However, in their analysis, the authors find that statistical Coulomb interactions limit the reduced brightness of even atomically sharp cold field emitters to $10^{11} \mathrm{~A} /\left(\mathrm{m}^{2} \mathrm{sr} \mathrm{V}\right)$ and regular tungsten cold field emitters to around $2 \times 10^{8} \mathrm{~A} /\left(\mathrm{m}^{2} \mathrm{sr} \mathrm{V}\right)$. The authors also find that for tip radii in the range from $5 \mathrm{~nm}$ to $1 \mu \mathrm{m}$, cold field emitters do not outperform larger Schottky (thermal field) emitters. Although this is applied to only one geometry, they expect that similar results will occur for most other cases due to a distinct difference in the behavior of different beam regimes. (C) 2010 American Vacuum Society. [DOI: 10.1116/1.3502642]
\end{abstract}

\section{INTRODUCTION}

The reduced brightness of field emitters according to Fowler-Nordheim theory ${ }^{1}$ can be extremely large, which should make electron microscopes significantly more powerful tools. ${ }^{1}$

However, despite this promise of high brightness, field emitters appear not to significantly outperform thermal field emitters, also known as Schottky emitters. The reduced brightness, which is constant for conservative fields, is in practice defined as

$$
B_{r}=\frac{I}{\pi \alpha^{2} \pi r_{\mathrm{vs}}^{2} V}
$$

where $\pi r_{\mathrm{vs}}^{2}$ is the virtual source size of the emitter, e.g., the area from which the current appears to come from, $\pi \alpha^{2}$ defines the solid angle, and $V$ is the beam potential.

The reduced brightness $B_{r}$ decides the amount of current $\left(I_{\text {probe }}=B_{r} \pi \alpha^{2} \pi r_{\text {probe }}^{2} V\right)$ in a probe of radius $r_{\text {probe }}$. For faster imaging times and even just to overcome signal-to-noise problems, the brightness $B_{r}$ sets the limit.

The brightness of field emitters is, according to standard theory (see Fig. 1), ${ }^{2-4}$ virtually limitless. Claims are made of $B_{r}$ as high as $10^{14} \mathrm{~A} /\left(\mathrm{m}^{2} \mathrm{sr} \mathrm{V}\right),{ }^{5,6}$ but — to the best of our knowledge - have never been measured. Some brightness measurements of field emitters are summarized in Ref. 7 Probably, the highest measured $B_{r}$ 's are 3 $\times 10^{9} \mathrm{~A} /\left(\mathrm{m}^{2} \mathrm{sr} \mathrm{V}\right)$ from a carbon nanotube ${ }^{8}$ and 4.6 $\times 10^{9} \mathrm{~A} /\left(\mathrm{m}^{2} \mathrm{sr} \mathrm{V}\right)$ from a sharp tungsten tip. ${ }^{9}$ In 2001 , van Veen et al. measured the brightness of a Schottky emitter and found experimentally a maximum $B_{r}$ of 2 $\times 10^{9} \mathrm{~A} /\left(\mathrm{m}^{2} \mathrm{sr} \mathrm{V}\right) .{ }^{10}$ They found that at high fields - and therefore at high $B_{r}$ - it is less than theoretically antici-

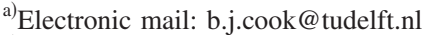

${ }^{1)}$ Cold field emitters can have much lower energy spreads than Schottky (thermal field) emitters. In cases where the dominant contribution to the probe size is a chromatic aberration, then a cold field emitter can outperform a Schottky emitter.
}

pated. This could be due to many reasons. For example, vibrations/stray magnetic fields can increase the virtual source size. These will not change with voltage, only acting as a constant blur to the virtual source size $r_{\mathrm{vs}}$. An error in the emission model is possible, but this is a well researched, mature field. The most likely conclusion is that brightness $B_{r}$ is not constant due to electron-electron interactions, which are not the same for every electron. Hagen et al. also measured the brightness of a liquid metal ion source. ${ }^{11}$ They concluded that its brightness was lowered due to statistical Coulomb interactions. These interactions cause the trajectories of the electrons to be perturbed. The emission process is governed by a transmission probability and the statistical distribution function of Fermi and Dirac. Therefore, the exact location of the electrons in phase-space (position and velocity) is unknown. This means that the electron-electron forces are also unknown. The trajectory of an electron through such a field can only be described statistically, introducing a blurring term to the virtual source size $r_{\mathrm{vs}}$, and thus, reducing the brightness $B_{r}$.

This blurring is likely to be more significant at greater particle densities, and therefore, at higher current densities $\left[J=I /\left(\pi r_{\mathrm{vs}}^{2}\right)\right]$. Due to Eq. (1), this blurring effect increases as the brightness $B_{r}$ increases. This blurring effect is also influenced by the particle interaction time. So at higher beam voltages, less interactions should occur. Current densities at the source are normally high and the potential is close to zero. Therefore, most Coulomb interactions occur in this region, and they should always be calculated.

Electron-electron interactions are often calculated by Monte Carlo simulations. However, Monte Carlo simulations provide little physical insight, are time consuming, and must be recalculated for every geometry change. We are interested in analytical equations describing the amount of statistical blurring. We, therefore, use the extended two-particle approximation developed by Jansen. ${ }^{12,13}$ Although it is an approximation, it has been experimentally validated ${ }^{10,11}$ and appears to be accurate within the experimental error. ${ }^{10}$ 


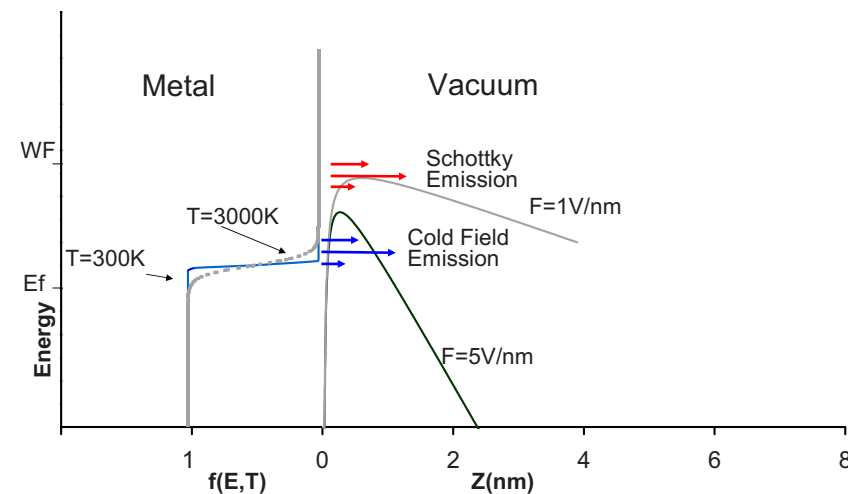

FIG. 1. (Color online) Schematic figure of cold field emission and thermal emission. On the left, electrons in the metal are distributed according to Fermi-Dirac statistics at $T=300 \mathrm{~K}$ and $T=3000 \mathrm{~K}$. On the right, an imagecharge barrier is shown at both fields of 1 and $5 \mathrm{~V} / \mathrm{nm}$. Due to the small width of the $5 \mathrm{~V} / \mathrm{nm}$ barrier, electrons near the Fermi energy $E_{f}$ can tunnel to the vacuum (indicated by the lower arrows). For the $1 \mathrm{~V} / \mathrm{nm}$ barrier, there is little tunneling near the Fermi energy and only thermally excited electrons escape. The electrons either tunnel through the top of the barrier or escape directly due to the Schottky lowered work function. In this model, electronelectron interactions are ignored. Emission is assumed to be from a semiinfinite solid and Nottingham heating, which may cause tip melting, is ignored (Ref. 20). Hence, the current density $J$ and reduced brightness $B_{r}$ can increase almost without limit.

The objectives of this work are to calculate the trajectory displacement due to statistical Coulomb interactions in the gun area and to determine their influence on the brightness of cold field emitters. The amount and distribution of field emitted current are directly dependent on the gun geometry and voltages, which determine the field at the emitter surface. In our model, we first calculate the field on the emitter surface and then solve the emission equations. This is often not done, and currents are arbitrarily chosen.

The reader should note that statistical interactions are separate from space-charge, where the latter creates a conservative field and smooths out the charge. It does not consider the discrete nature of the individual electrons. The interested reader is referred to Ref. 13 .

\section{THEORY}

The extended two-particle method splits the longitudinal and lateral electron interactions. We consider only $B_{r}$ reduction, known as trajectory displacement coming from the lateral interactions. The method is valid for most regimes of interest in imaging and lithography machines.

It is assumed that the particle's positions and velocities are uncorrelated. Then, it approximates the many-body problem to a sum of two-body interactions on a test particle traveling along the axis. This ensures that the space-charge effect is automatically canceled out. It has been shown that an onaxis particle is representative of the beam as a whole. ${ }^{12}$ The beam is split up into segments with constant voltage and either a conical or cylindrical beam envelope. Although exact analytical solutions do not exist, Jansen found different regimes depending on particle density and beam geometry. For these regions, more practical analytical solutions can be

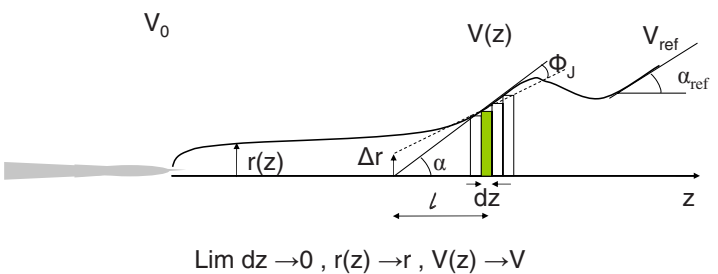

FIG. 2. (Color online) From Left to right: The needle cathode, half the beam-envelope (curved line) with beam radius $r(z)$ at potential $V(z)$ traveling along the $z$-axis to our reference point with voltage $V_{\text {ref }}$. The beam-space is split into small segments $d z$ for which we assume that $V(z)$ and $r(z)$ are constant. The angular displacement $\phi_{J}$ is found from Eq. (5). The trajectory total displacement is found by adding the displacement $\Delta r$ at each slice to find $\Delta r_{\text {traj }}$ as given by Eq. (6).

found by fitting and approximating. In order to find the trajectory displacement, we first calculate the angular displacements; we give the answer as a FW50\% (full width containing $50 \%$ of the distribution) of the distribution. The relevant equations and their regimes are shown below. For the Gaussian regime,

$$
\phi_{G}=0.1667 \frac{m^{1 / 4} e^{1 / 12}}{\epsilon_{0}^{5 / 6}} \frac{(J \pi)^{1 / 2} L^{2 / 3}}{V^{13 / 12}} .
$$

For the Holtsmarkian regime,

$$
\phi_{H}=0.128 \frac{m^{1 / 3}}{\epsilon_{0}} \frac{(J \pi)^{2 / 3} L}{V^{4 / 3}} .
$$

For the pencil beam regime,

$$
\phi_{p}=0.429 \frac{m^{3 / 2}}{\epsilon_{0} e^{7 / 2}} \frac{(J \pi)^{3} r^{7} L}{V^{5 / 2}},
$$

where $m$ is the electron mass, $\epsilon_{0}$ is the permittivity of free space, and $L$ is the interaction length. The above equations are valid for large particle densities (Gaussian), low particle densities (Holtsmarkian), and low particle densities with a narrow beam (pencil beam), respectively. These equations are only valid for a cylindrical beam segment at a constant potential. For an electron gun with varying potential and beam size, we must apply the slice method. The region to be calculated is divided into small segments over which the voltage and beam size is assumed to remain constant (see Fig. 2).

Equations (2)-(4) have been interpolated by Jiang et al., so these can be used without knowledge of the regime. ${ }^{14}$ The slice method requires the angular displacement per meter, given by

$$
\phi_{J}=\left[\frac{T_{1} D_{\lambda}^{18 / 7} D_{r}^{6} I^{18 / 7} r^{6 / 7} V^{-15 / 7}}{T_{4}+T_{2}^{1 / 7} D_{r}^{6} D_{\lambda}^{2} I^{2} r^{2} V^{-1}}\right]^{7 / 6},
$$

where $D_{\lambda}=m^{(1 / 2)} /\left(\pi 2^{(7 / 2)} \epsilon_{0} e^{(1 / 2)}\right)$ and $D_{r}=\left(2 \epsilon_{0} \pi / e\right)^{1 / 3}$. The constants are $T_{1}=4.618 \times 10^{-2}, T_{2}=2.041 \times 10^{5}$, and $T_{4}$ $=6.250 \times 10^{-2}$. In the interpolation, the Gaussian regime disappears to zero. One must be careful for large particle densities, as a Gaussian distribution can still develop. Therefore, we are limited to either a pencil beam or a Holtsmarkian beam. 


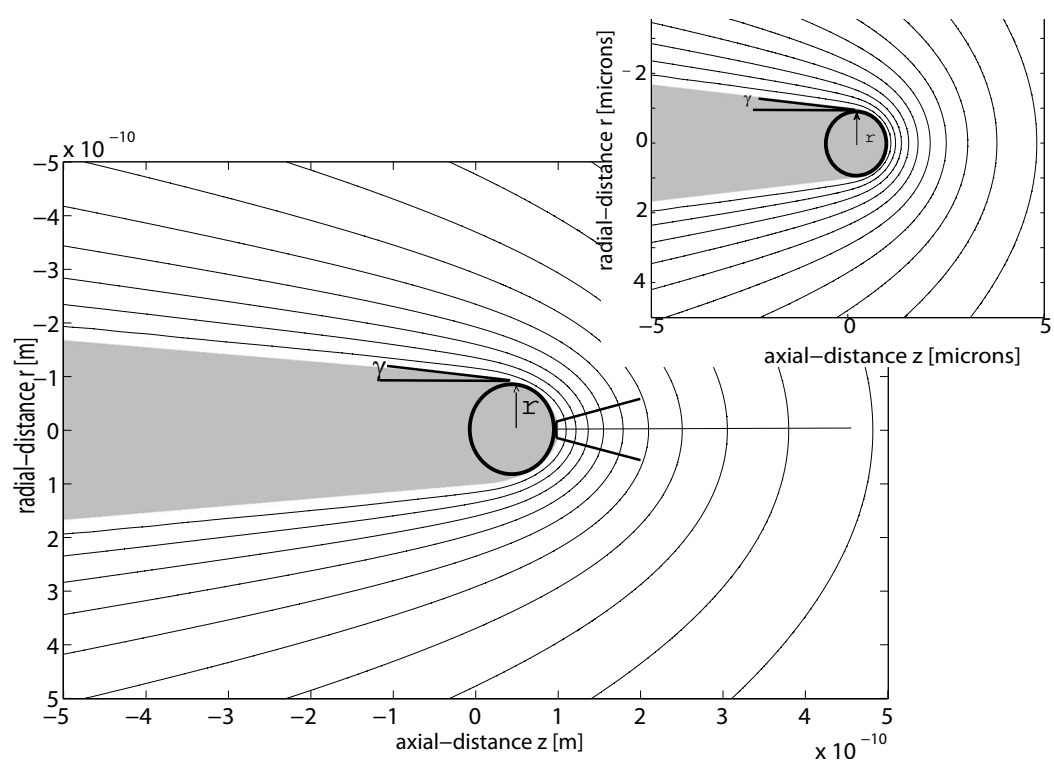

FIG. 3. Close-up of an atomically sharp tip with equipotential lines. The inset is a close-up of a $1 \mu \mathrm{m}$ tip. The tip scales with regard to the tip radius $r$, having constant cone angle $\gamma$. The field around each tip is smooth and scales as expected. The black cone coming from the end of the field emission tip indicates where we allow the current to come from. This may be arbitrary, but allowing extra current only increases Coulomb interactions.

From the slice method, the trajectory displacement is calculated by

$$
r_{\text {traj }}=\int_{z} \frac{l(z)}{M(z)} \phi_{J}(r(z), V(z), I) d z .
$$

We find $l(z)$ from $r(z) / \alpha(z)$, and $M(z)$ is the magnification $\left(=\left(\alpha_{\text {ref }} / \alpha(z)\right) \sqrt{\left(V_{\text {ref }} / V(z)\right)}\right) \cdot V_{\text {ref }}$ is the voltage at the reference plane in the field-free region at $1.2 \mathrm{~mm}$. The virtual source radius $r_{\mathrm{vs}}$ is defined looking back from this plane.

The trajectory displacement translates as a blurring of the virtual source size, and so we modify $B_{r}$ as

$$
B_{r(e-e)}=B_{r}\left(\frac{r_{\mathrm{vs}}^{2}}{\left(r_{\mathrm{vs}}^{2}+r_{\text {traj }}^{2}\right)}\right) \text {. }
$$

We use a Gaussian addition rule, as it is generally a good first approximation and as in the case of Ref. 10, it seemed to work. However, for more accurate results, there are better addition laws, see, for example, Ref. 15.

\section{METHOD}

We have developed our own suite of software at Delft to investigate Coulomb interactions. This section describes the steps taken by the software to calculate the trajectory displacement.

The initial step is to draw the gun and assign voltages to each section. The drawing is implemented through our vector graphics routine. Each section is defined from primitive shapes (squares, circles, triangles, etc.). This allows us to define more complex geometries such as the needle cathode given by Fig. 3 .

The Poisson solver calculates the electric field to a user defined precision; see Fig. 4 for the gun design we used. Only the tip radius was varied. Due to the nature of the problem, we have used spherical coordinates and cylindrical symmetry. The small needle tip is at the center (where the grid and field lines are most dense). This is similar to spherical coordinate with increasing mesh size developed by Kang et al. ${ }^{16}$

With the potential field known, the outer electrons are ray-traced through the system. This can again be done to user defined precision. Stability criteria are checked before raytracing starts. In general, a step size smaller than $10^{-16} \mathrm{~s}$ is used. The electrons are launched immediately in front of the tip with no velocity at a height of one-fourth the tip radius (see Fig. 3). This is somewhat unrealistic, but only serves to underestimate the Coulomb effect by limiting the current. Experimental evidence suggests that we reached the pencil

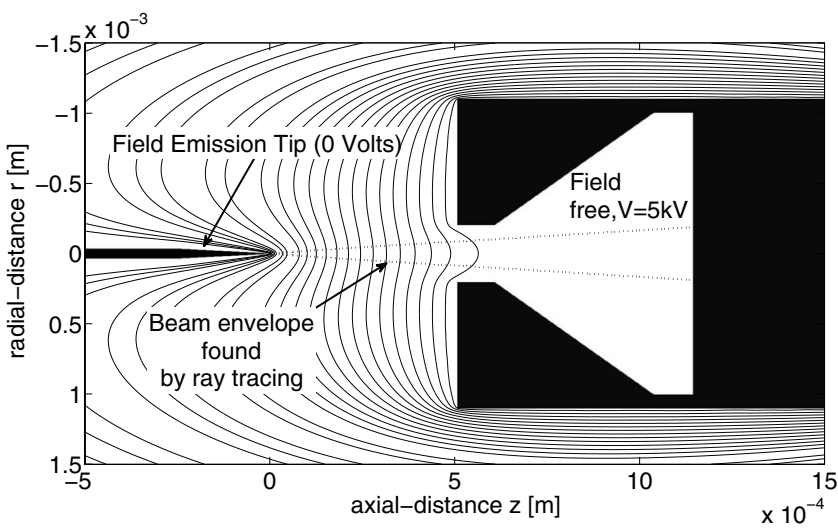

FIG. 4. Schematic figure of the gun geometry. As indicated, the field emission tip (for this figure, $r=1 \mu \mathrm{m}$ ) is at ground potential. The extractor, for this example, is at $5 \mathrm{kV}$. There is a field-free zone after the extractor. The beam-envelope is shown with a dotted black line. The trajectory appears straight on this scale. 


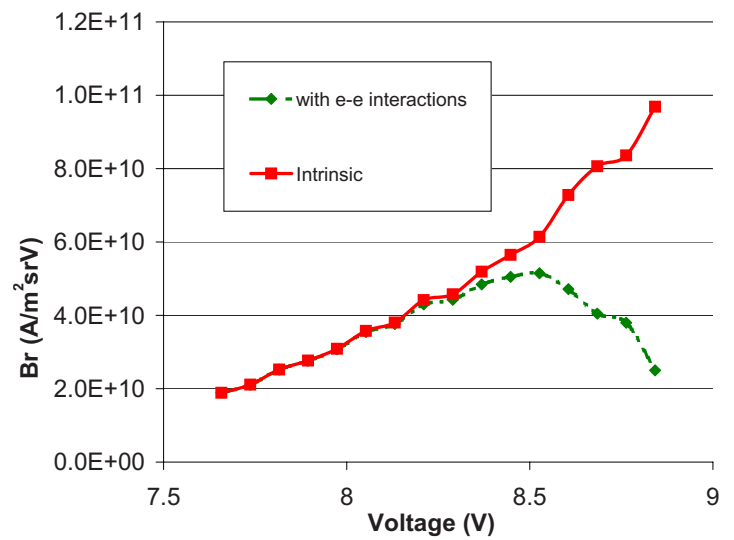

FIG. 5. (Color online) Reduced brightness of an atomically sharp tip (100 p.m. radius) as a function of extractor voltage. The upper solid line with $\square \mathrm{s}$ is the intrinsic $B_{r}$ without Coulomb interactions, the lower dot-dashed line with $\diamond \mathrm{s}$ includes Coulomb interactions. Initially, the Coulomb interactions have very little effect on the brightness, but once $r_{\text {traj }}$ becomes comparable to the $r_{\mathrm{vs}}$, the effect of the trajectory displacement is felt and we quickly reach a maximum at $8.5 \mathrm{~V}$ and $B_{r}=5 \times 10^{10} \mathrm{~A} /\left(\mathrm{m}^{2} \mathrm{sr} \mathrm{V}\right)$.

beam regime (see Sec. IV for further discussion). This will not happen if the beam is too wide. Also, electrons traveling with large angles will do little.

We take the electric field at the surface of the tip. If the field allows tunneling $(>0.1 \mathrm{~V} / \mathrm{nm})$, we numerically solve Schrodinger's equation with Numerov's method (see, for example, Ref. 17). Similar results are obtained by using the Runge-Kutta method. ${ }^{4}$ Otherwise, a step barrier with Schottky lowered work function $\left[\Delta W=\sqrt{F e^{3} /\left(4 \pi \epsilon_{0}\right)}\right]$ is applied, where $F$ is the electric field. ${ }^{3}$ The emission, in terms of current density and brightness, is solved as per Ref. 4. This is valid for almost every situation of interest, including all combinations of thermal, field, and photoemission.

Next, the slice method is applied to calculate the trajectory displacement given by Eq. (6). We verify the linear particle density $(=I e / \sqrt{2} \mathrm{eV} / \mathrm{m})$ and the beam size to ensure that we do not work outside the limitations of our equations.

Variables are saved and the whole procedure is repeated with the required variable changed.

\section{RESULTS AND DISCUSSION}

After verifying that our software reproduces previously found results, ${ }^{10,18,19}$ we were intrigued to investigate how tip size would affect the trajectory displacement. We started with the atomically sharp tip in Fig. 3. Although our atomic tip may be impossible to achieve in reality, in our simulation they are simple to generate and a valid shape to start investigating fundamental limits. The intrinsic reduced brightness $B_{r}$ of a (cold) field emission tip is determined by the field and the work function of the emitter. Tungsten is widely used, and a work function of $4.3 \mathrm{eV}$ is reasonable. To discover how $B_{r(e-e)}$ varied with field, we changed the extractor voltage, creating Fig. 5. Our expectation was that as the extractor voltage increases, so would $B_{r}$ and $B_{r(e-e)}$. From the experimental results mentioned in Sec. I, we expected that at around $10^{9} \mathrm{~A} /\left(\mathrm{m}^{2} \mathrm{sr} \mathrm{V}\right), B_{r(e-e)}$ would drop slowly away

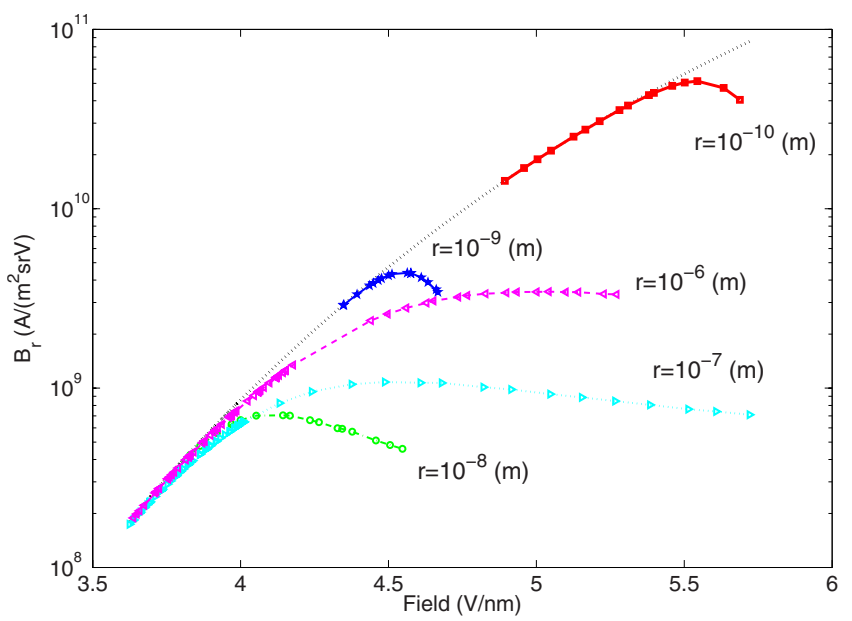

FIG. 6. (Color online) Brightness as a function of field for various source radii, with and without Coulomb interactions. Dotted line without marker is intrinsic $B_{r}$ without Coulomb interactions. Solid line with $\square$ s is for a source radius of $10^{-10} \mathrm{~m}$ including Coulomb interactions. Solid line with $\star \mathrm{s}$ is a source radius of $10^{-9} \mathrm{~m}$ including Coulomb interactions. Dashed line with $\nabla_{\mathrm{s}}$ is a source radius of $10^{-6} \mathrm{~m}$. Dotted line with $\nabla_{\mathrm{s}}$ is a source radius of $10^{-7} \mathrm{~m}$. Dashed line with $\bigcirc \mathrm{s}$ is a source radius of $10^{-8} \mathrm{~m}$. The two smallest radii have the sharpest cutoff/clearest maximum, and as the emitter radius increases, so the gradient around the maximum decreases. However, the most striking feature of this graph is that the emitter with the $1 \mu \mathrm{m}$ radius has a brightness well above that of the 100 and $10 \mathrm{~nm}$ emitters.

from $B_{r}$. We thought this should be slow due to the inverse proportionality of $r_{\text {traj }}$ to $V$. Hence, we were surprised to see it quickly reach a maximum at $5 \times 10^{10} \mathrm{~A} /\left(\mathrm{m}^{2} \mathrm{sr} \mathrm{V}\right)$ and fall away. For all points on the graph, we are within the Holtsmark/pencil beam regime. Probably mostly influenced by the pencil beam. The behavior stems from the $I^{3} / V^{2.5}$ in the pencil beam regime and the exponential dependence of $I$ with surface field.

To see the trend in the maximum brightness, we calculated $B_{r(e-e)}$ for several other tip radii. Results are plotted in Fig. 6. The maxima do not follow directly the tip radius. We expected the brightest would be the atomically sharp tip, and the least bright would be the $1 \mu \mathrm{m}$ tip. Our logic was based on traditional wisdom that sharp tips are best. By reducing the total number of electrons, the Coulomb interactions should decrease. However, this trend is bucked. The bluntest tip has almost comparable brightness to a $1 \mathrm{~nm}$ tip. We wanted to check whether this was a computational error. We calculated analytically, using Eqs. (3) and (4), the trajectory displacement for a cylindrical beam segment with constant radius and voltage. The field on the tip is determined by $(V / L)$ with $L=0.5 \mathrm{~mm}$ (see inset in Fig. 7), and the intrinsic brightness $B_{r}$ is calculated as $B_{r}=e J / \pi d$, where $J$ $=\left(4 \pi m e / h^{3}\right) d^{2} \exp (-W / d), d=e h F / 4 \pi \sqrt{(2 m W)}, W$ is the material's work function, and $h$ is Planck's constant, see Ref. 2 or 3 . According to Ref. 13, we may add the contributions to the virtual source radius from the different regimes as

$$
\frac{1}{S_{\text {total }}}=\frac{1}{S_{\text {Holtsmark }}}+\frac{1}{S_{\text {pencil }}},
$$

where $S_{\text {total }}$ is the increase in virtual source radius due to trajectory displacement. $S_{\text {total }}$ is made up of two components: 


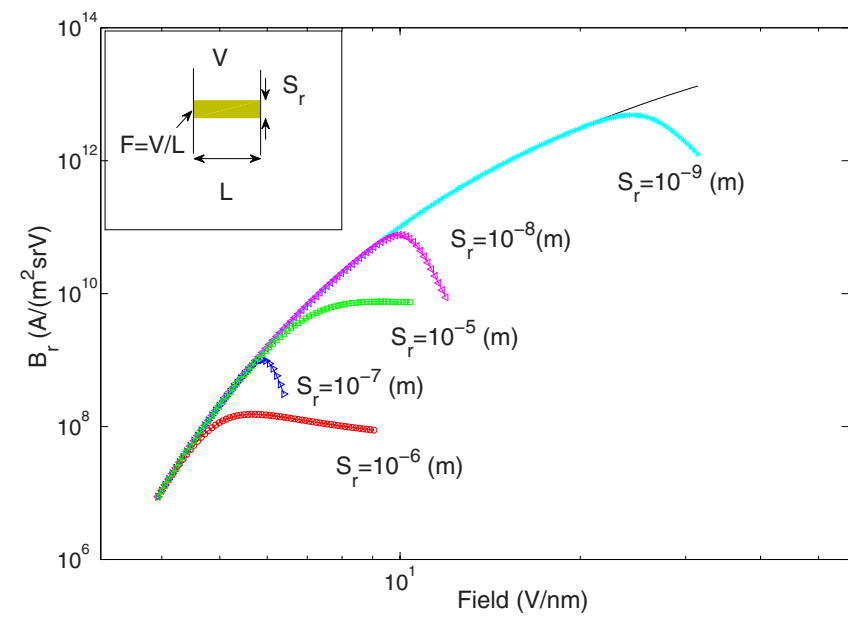

FIG. 7. (Color online) Analytical plot of brightness vs field for different tip radii $\left(S_{r}\right)$. Inset shows the layout used for this experiment. $V$ is not the extractor voltage but the potential of the beam from the start. In other words, the beam has a constant velocity. The emission current is calculated from a pseudofield given by $(V / L)$. The solid line without markers is the intrinsic brightness $B_{r}$. The line with $\star \mathrm{s}$ is for a $1 \mathrm{~nm}$ source with Coulomb interactions. $\triangleright$ is $10 \mathrm{~nm}$ with Coulomb interactions. $\square$ is $10 \mu \mathrm{m}$ with Coulomb interactions. $\triangleleft$ is $100 \mathrm{~nm}$ with Coulomb interactions. $\bigcirc$ is $1 \mu \mathrm{m}$ with Coulomb interactions. Just like the numerically produced results, the blunter tip has a larger brightness than sharper tips $(1 \mu \mathrm{m}$ and $100 \mathrm{~nm})$. This demonstrates that the effect is not due to miscalculation of fields or ray-tracing.

$S_{\text {Holtsmark }}$, the trajectory displacement in the Holtsmark regime, and $S_{\text {pencil }}$ from the pencil beam regime. In essence, Eq. (8) will pick out the smallest contribution to the virtual source. The result is shown in Fig. 7.

The analytical work is similar to the fully numerical result, so we are certain we have calculated it correctly. This analytical approach can never be as accurate as the numerical approach because, around a sharp tip, the potential and beam radius change very rapidly. However, it does help us to see that the observed behavior stems from competition between the Holtsmark and pencil beam regimes.

To better see the dependence of $B_{r(e-e)}$, we plotted separately the contributions of the pencil beam and the Holtsmark regimes in Fig. 8. The contribution of the pencil beam regime to the virtual source radius $\left(S_{\text {pencil }}\right)$ scales with $S_{r}$ the source radius, the brightness of a pencil beam is

$$
B_{r(e-e)}=B_{r} \frac{S_{r}}{S_{r}^{2}+\left(\beta_{p b}\left((J)^{3} S_{r}^{7} L^{2} / V^{5 / 2}\right)\right)^{2}},
$$

where $\beta_{p b}=0.429\left(m^{3 / 2} \pi^{3} / \epsilon_{0} e^{7 / 2}\right)$, and we have substituted $S_{\text {pencil }}$ for Eq. (4) multiplying by $L$ to get the displacement at the source.

In the Holtsmark regime, the trajectory displacement is constant for all beam radii (in this example, current density does not change with source radius), so $S_{\text {Holtsmark }}$ remains constant.

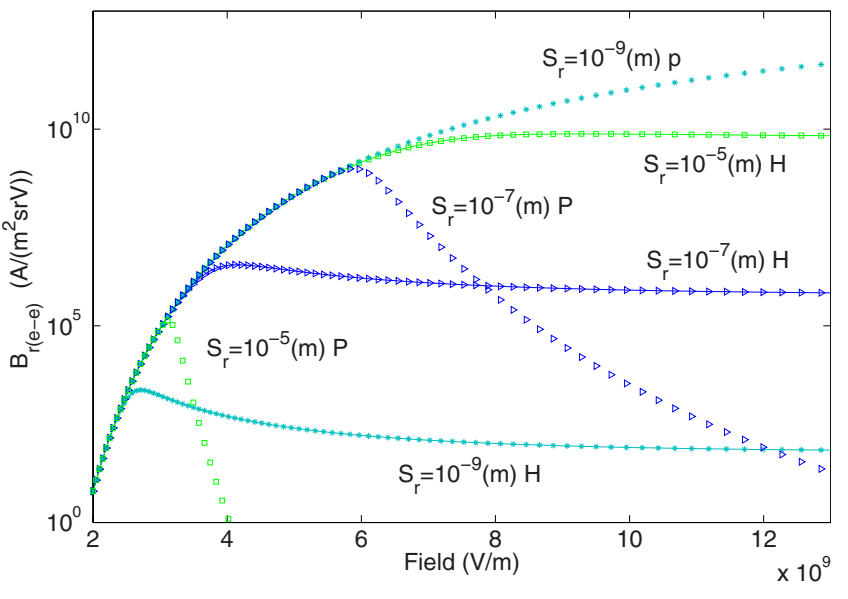

FIG. 8. (Color online) Reduced brightness with Holtsmark and pencil beam contributions shown separately labeled $H$ and $P$, respectively. Pencil beam has no line and Holtsmark has a solid line. ${ }^{*} \mathrm{~s}$ are for $S_{r}=10^{-9} \mathrm{~m}, \nabla \mathrm{s}_{\mathrm{s}}$ for $S_{r}=10^{-7} \mathrm{~m}$, and $\square \mathrm{s}$ for $S_{r}=10^{-5} \mathrm{~m}$. We can see that the pencil beam scales with $S_{r} \mathrm{~m}$ and the Holtsmark regime follows inversely.

$$
B_{r(e-e)}=B_{r} \frac{S_{r}^{2}}{S_{r}^{2}+\left(\beta_{H}\left((J)^{2 / 3} L^{2} / V^{4 / 3}\right)\right)^{2}},
$$

where $\beta_{H}=0.128\left(m^{1 / 3} \pi^{2 / 3} / \epsilon_{0}\right)$ and we have substituted $S_{\text {Holtsmark }}$ for Eq. (3) multiplied by $L$.

As $S_{r}$ increases, the effect of the constant $S_{\text {Holtsmark }}$ decreases. This means that if we are in the Holtsmark regime or, in fact, even the Gaussian regime [see Eq. (2)], bigger cathodes are less affected by Coulomb interactions than smaller ones. However, other practical issues involved with generating the fields give difficulties.

For our larger tips, we must be careful that we are not in the Gaussian regime; this is not suited to the slice method. At the maximum brightness, we are close. However, when the brightness drops to $90 \%$ of its intrinsic value $\left(B_{r(e-e)}\right.$ $=0.9 B_{r}$ ), we are still valid for all tip sizes investigated. To summarize our findings, we plotted the value of $B_{r}$ for $B_{r(e-e)}=0.9 B_{r}$ for different tip radii in Fig. 9. Included also are a Schottky tip and photoassisted Schottky tip (PHAST)

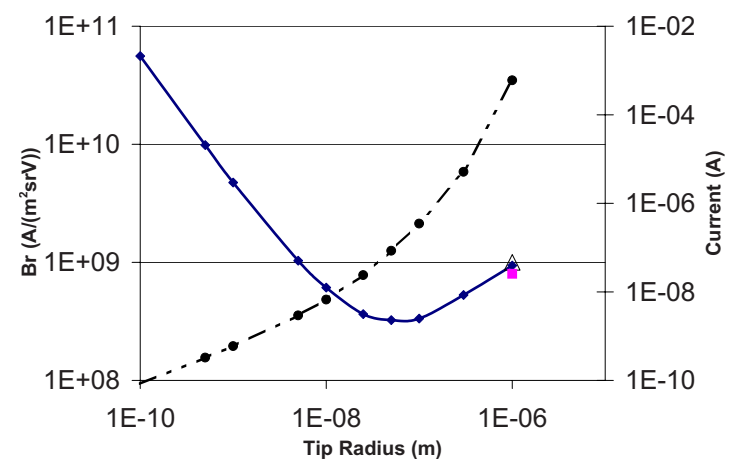

FIG. 9. (Color online) Solid line with $\diamond \mathrm{s}$ brightness at $10 \%$ reduction $\left(B_{r(e-e)}=0.9 B_{r}\right)$, as a function of tip radius, for cold field emitters. Dashed line with $O s$ is current at $10 \%$ reduction (right axis). The $\triangle$ and $\square$ are approximate values for a Schottky emitter and PHAST (see Sec. IV). It is important to note that the minimum at $10 \mathrm{~nm}$ is due to the competition between the pencil beam regime and the Holtsmark regime. 
taken from Refs. 19 and 18, respectively. They are from different setups with only similar parameters, so care must be taken when making comparisons; however, they are brighter than the $100 \mathrm{~nm}$ tips we simulated. It is worth noting that the Schottky tips in Refs. 18 and 19 have $T=1800 \mathrm{~K}$, and $W$ $=2.8 \mathrm{eV}$. Due to the high temperature, it has good stability (the surface remains free of dirt) and tends to have a longer lifetime than cold field emitters. Thus, although intrinsically $B_{r}$ calculation makes it appear less bright when Coulomb effects are taken into account, it performs as well as the cold field emitter. We have also included the current used in the calculation for reference. All tips would be suitable for imaging, though perhaps the smaller ones would not be suitable for lithography due to the low current.

\section{CONCLUSIONS}

We find that Coulomb interactions significantly limit $B_{r}$ in cold field emitters. Claims of $B_{r}$ greater than $10^{12} \mathrm{~A} /\left(\mathrm{m}^{2} \mathrm{sr} \mathrm{V}\right)$ should be viewed with caution; we expect that most field emitters have $B_{r}$ between $10^{8}$ and $10^{9} \mathrm{~A} /\left(\mathrm{m}^{2} \mathrm{sr} \mathrm{V}\right)$, with only a few working at their true potential. Cold field emitters may have a lower energy spread than Schottky emitters, and therefore, could outperform them in imaging. We have not considered this. Our conclusion is, that when designing electron guns for high brightness, with cold field emitters, Coulomb interactions must be taken into account. Otherwise, the extra hassle (ultrahigh vacuum, regular flash cleaning, short lifetime) associated with cold field emission will be wasted.

\section{ACKNOWLEDGMENTS}

This work is funded by "Stichting voor Fundamenteel Onderzoek der Materie" (FOM), which is financially sup- ported by the "Nederlandse Organisatie voor Wetenschappelijk On-derzoek" (NWO). The program is cofinanced by FEI Company.

${ }^{1}$ R. H. Fowler and L. Nordheim, Proc. R. Soc. London, Ser. A 119, 173 (1928).

${ }^{2}$ H. Shimoyama and S. Maruse, Ultramicroscopy 15, 239 (1984).

${ }^{3}$ E. Kasper and P. W. Hawkes, Principles of Electron Optics (Academic, London, England, 2009), Vol. 2, Chap. 44, , p. 918.

${ }^{4}$ B. Cook, M. S. Bronsgeest, C. W. Hagen, and P. Kruit, Ultramicroscopy 109, 403 (2009).

${ }^{5}$ C. A. Brau, Nucl. Instrum. Methods Phys. Res. A 407, 1 (1998).

${ }^{6}$ J. C. H. Spence, T. Vecchione, U. Weierstall, and G. Hembree, Microsc. Microanal. 11, 480 (2005).

${ }^{7}$ M. J. Fransen, M. H. F. Overwijk, and P. Kruit, Appl. Surf. Sci. 146, 357 (1999).

${ }^{8}$ N. de Jonge, Y. Lamy, K. Schoots, and T. H. Oosterkamp, Nature (London) 420, 393 (2002).

${ }^{9}$ J. C. H. Spence, W. Qian, and M. P. Silverman, J. Vac. Sci. Technol. A 12, 542 (1994).

${ }^{10}$ A. H. V. van Veen, C. W. Hagen, J. E. Barth, and P. Kruit, J. Vac. Sci. Technol. B 19, 2038 (2001).

${ }^{11}$ C. W. Hagen, E. Fokkema, and P. Kruit, J. Vac. Sci. Technol. B 26, 2091 (2008).

${ }^{12}$ G. H. Jansen, Coulomb Interactions in Particle Beams, Suppliment 21 of the Series "Advances in Electronics and Electron Physics" (Academic, New York, 1990), Chap. 5, p. 91

${ }^{13} \mathrm{G}$. H. Jansen and P. Kruit, Handbook of charged particle optics (CRC, Boca Raton, FL, 2009), Chap. 7, Vol. 2, p. 349.

${ }^{14}$ J. Bi, X. R. Jiang, and P. Kruit, Microelectron. Eng. 35, 439 (1997).

${ }^{15}$ M. S. Bronsgeest, J. E. Barth, G. A. Schwind, L. W. Swanson, and P. Kruit, J. Vac. Sci. Technol. B 25, 2049 (2007).

${ }^{16}$ N. K. Kang, J. Orloff, L. W. Swanson, and D. Tuggle, J. Vac. Sci. Technol. 19, 1077 (1981).

${ }^{17}$ J. Thijssen, Computational Physics (Cambridge University Press, Cambridge, England, 2007), Vol. 2, p. 21.

${ }^{18}$ B. Cook, M. S. Bronsgeest, C. W. Hagen, and P. Kruit, Proceedings of IVESC-2008 (unpublished).

${ }^{19}$ M. S. Bronsgeest, Ph.D. thesis, Delft University of Technology, 2009.

${ }^{20}$ W. B. Nottingham, Phys. Rev. 59, 906 (1941). 